\author{
I50 Jahre \\ Wissen für die Zukunft \\ (48) Oldenbourg Verlag
}





\section{Physik II}

Elektrodynamik und Spezielle Relativitätstheorie

von

Prof. Dr. Klaus Dransfeld und

Prof. Dr.-Ing. Paul Kienle

7. aktualisierte Auflage

Oldenbourg Verlag München Wien 
Prof. Dr. Klaus Dransfeld hatte von 1981 bis zu seiner Emeritierung den Lehrstuhl für Physik an der Universität Konstanz inne. Zuvor war er an der University of California in Berkeley, an der TU München und von 1974-82 Direktor am Max-Planck-Institut für Festkörperphysik (zunächst am neuen deutsch-französischen Hochfeldmagnetlabor in Grenoble und später in Stuttgart). Seine Hauptarbeitsgebiete sind Ultraschall, tiefe Temperaturen, Nanotechnologie. 1989 erhielt er den Gentner-Kastler-Preis der Deutschen Physikalischen Gesellschaft und der Société Française de Physique sowie den Forschungspreis der Japan Society. Er ist Ehrendoktor der Universitäten Grenoble und Augsburg und Honorarprofessor der Universität Nanjing.

Prof. Dr.-Ing. Paul Kienle war von 2002-2004 Direktor des Stefan-Meyer-Instituts für subatomare Physik der Österreichischen Akademie der Wissenschaften, Wien. Frühere Stationen seiner Laufbahn: Inhaber des Lehrstuhls für Strahlen und Kernphysik an der TH Darmstadt (1963-65), anschließend Professor für Experimentalphysik an der TU München bis zur Emeritierung (1999). Aufbau des Beschleunigerlaboratorium der LMU und TU München mit Ulrich Meyer Berkhout (1965-71). Als Direktor der GSI Darmstadt Ausbau der Beschleuniger mit einem Synchrotron und Speicherring für schwere Ionen (1984-92). Humboldt-Preis der Republik Frankreich und Forschungspreis der Japan Society for the Promotion of Science. Wissenschaftliche Veröffentlichungen auf dem Gebiet der Kern und Teilchen Physik, Lehrbücher der Physik und andere wissenschaftliche Bücher.

Bibliografische Information der Deutschen Nationalbibliothek

Die Deutsche Nationalbibliothek verzeichnet diese Publikation in der Deutschen Nationalbibliografie; detaillierte bibliografische Daten sind im Internet über $<$ http://dnb.d-nb.de> abrufbar.

(c) 2008 Oldenbourg Wissenschaftsverlag $\mathrm{GmbH}$

Rosenheimer Straße 145, D-81671 München

Telefon: (089) 45051-0

oldenbourg.de

Das Werk einschließlich aller Abbildungen ist urheberrechtlich geschützt. Jede Verwertung außerhalb der Grenzen des Urheberrechtsgesetzes ist ohne Zustimmung des Verlages unzulässig und strafbar. Das gilt insbesondere für Vervielfältigungen, Übersetzungen, Mikroverfilmungen und die Einspeicherung und Bearbeitung in elektronischen Systemen.

Lektorat: Kathrin Mönch

Herstellung: Anna Grosser

Coverentwurf: Kochan \& Partner, München

Gedruckt auf säure- und chlorfreiem Papier

Druck: Grafik + Druck, München

Bindung: Thomas Buchbinderei $\mathrm{GmbH}$, Augsburg

ISBN 978-3-486-58598-8 